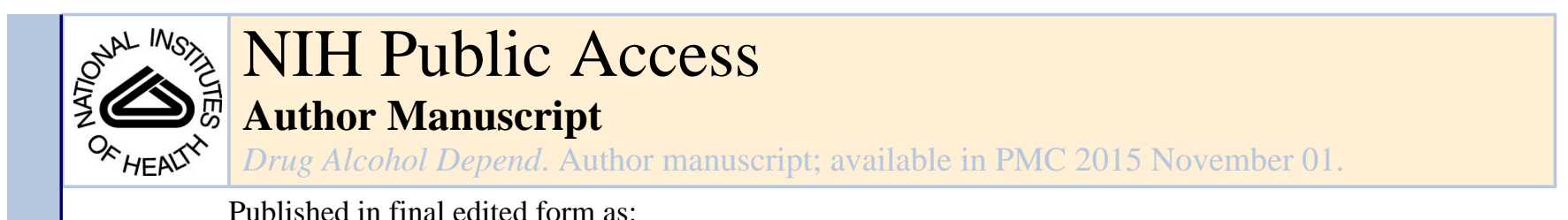

Published in final edited form as:

Drug Alcohol Depend. 2014 November 1; 144: 283-287. doi:10.1016/j.drugalcdep.2014.09.014.

\title{
Opioid Attentional Bias and Cue-Elicited Craving Predict Future Risk of Prescription Opioid Misuse Among Chronic Pain Patients ${ }^{*}$
}

\author{
Eric L. Garland and \\ Associate Director of Integrative Medicine, Huntsman Cancer Institute, University of Utah, 395 \\ South, 1500 East, Salt Lake City, UT 84112 \\ Matthew O. Howard \\ Frank Daniels Distinguished Professor, University of North Carolina - Chapel Hill, Tate Turner \\ Kuralt Bldg. 325 Pittsboro St \#3550, Chapel Hill, NC 27599
}

\begin{abstract}
Background-Some chronic pain patients receiving long-term opioid analgesic pharmacotherapy are at risk for misusing opioids. Like other addictive behaviors, risk of opioid misuse may be signaled by an attentional bias (AB) towards drug-related cues. The purpose of this study was to examine opioid $\mathrm{AB}$ as a potential predictor of opioid misuse among chronic pain patients following behavioral treatment.
\end{abstract}

Methods-Chronic pain patients taking long-term opioid analgesics $(\mathrm{N}=47)$ completed a dot probe task designed to assess opioid $\mathrm{AB}$, as well as self-report measures of opioid misuse and pain severity, and then participated in behavioral treatment. Regression analyses examined opioid $A B$ and cue-elicited craving as predictors of opioid misuse at 3-months posttreatment follow-up.

Results-Patients who scored high on a measure of opioid misuse risk following treatment exhibited significantly greater opioid $\mathrm{AB}$ scores than patients at low risk for opioid misuse. Opioid AB for 200 ms cues and cue-elicited craving significantly predicted opioid misuse risk 20 weeks later, even after controlling for pre-treatment opioid dependence diagnosis, opioid misuse, and pain severity (Model $R^{2}=.50$ ).

Conclusion-Biased initial attentional orienting to prescription opioid cues and cue-elicited craving may reliably signal future opioid misuse risk following treatment. These measures may therefore provide potential prognostic indicators of treatment outcome.

\footnotetext{
* Supplementary material can be found by accessing the online version of this paper at http://dx.doi.org and by entering doi:... (C) 2014 Elsevier Ltd. All rights reserved.

Corresponding author: Eric L. Garland, 395 South, 1500 East, University of Utah, Salt Lake City, UT 84112, elgarlan@ gmail.com. Contributors: ELG designed the study and wrote the protocol. ELG and MOH managed the literature searches and summaries of previous related work. ELG undertook the statistical analysis, and ELG and MOH wrote the first draft of the manuscript. All authors contributed to and have approved the final manuscript.

Conflict of Interest: Neither ELG nor MOH have any conflicts of interest to disclose.

Publisher's Disclaimer: This is a PDF file of an unedited manuscript that has been accepted for publication. As a service to our customers we are providing this early version of the manuscript. The manuscript will undergo copyediting, typesetting, and review of the resulting proof before it is published in its final citable form. Please note that during the production process errors may be discovered which could affect the content, and all legal disclaimers that apply to the journal pertain.
} 


\section{Keywords}

opioid misuse; chronic pain; attentional bias; cue-reactivity; implicit cognition; dot probe

\section{INTRODUCTION}

Chronic pain conditions are commonly treated with prescription opioids, which may relieve pain by targeting the descending pain modulatory system (Besson, 1999), decreasing somatosensory and thalamic activation (Wagner et al., 2007; Wise et al., 2002), modifying amygdala function (Oertel et al., 2007), and altering neurotransmission in spinal dorsal horn (Le Bars et al., 1980; Yaksh, 1985, 1981). In addition to analgesia, opioid administration stimulates dopamine release from the ventral tegmental area to the nucleus accumbens, facilitating opioid-related reinforcement learning (Shippenberg et al., 1993). Through this mechanism, opioids and opioid-related cues are imbued with incentive salience and come to signal pain relief and reward (Becker et al., 2012; Robinson and Berridge, 2001). Opioidinduced reward learning may be one key process underpinning the transition from medically authorized opioid use to opioid misuse and addiction (Garland et al., 2013a).

While a majority of chronic pain patients take opioids as prescribed, a substantial subset of patients are at risk for escalating from appropriate opioid use to misuse. A systematic review concluded that $11.5 \%$ of chronic pain patients evidenced signs of opioid misuse including self-medication of negative affect with opioids and unauthorized dose escalation (Fishbain et al., 2007). It is important to ascertain which patients are at risk for opioid misuse. Yet, these determinations are difficult because many chronic pain patients seek opioids for pain relief. Inadequately treated pain and physiological tolerance can result in opioid-seeking behaviors and opioid dose escalation - a pattern termed pseudoaddiction (Wilson, 2007). Fear of being stigmatized, legally penalized, or deprived of medication by health care providers may lead some patients to conceal opioid misuse. As such, cognitive tasks that do not rely on patient self-report may be useful in identifying individuals at risk for opioid misuse.

Cognitive tasks may have prognostic value in detection and prediction of opioid misuse risk because misuse of opioids, like other addictive behaviors, may involve automatic cognitive processes that organize and drive compulsive drug taking (Stacy and Wiers, 2010).

Recurrent substance use establishes automatic drug-use action schemas that compel consumption of the substance through automatized sequences of stimulus-bound, contextdependent behavior, including the biasing of attention towards substance-relevant stimuli (Pierce and Vanderschuren, 2010; Tiffany, 1990). Insofar as cues associated with past drug use are motivationally salient for habitual drug users, they capture attention, which amplifies their motivational salience (Franken, 2003). This phenomenon--addiction attentional bias (AB)--is evidenced on dot probe tasks by shorter reaction times (RT) to probes replacing drug-related images relative to probes replacing neutral images (Field and Cox, 2008). Addiction AB is associated with craving (Field et al., 2009) and has been reported to predict relapse following addiction treatment (e.g., Cox et al., 2002; Garland et al., 2012a).

$\mathrm{AB}$ toward heroin-related stimuli has been observed among heroin dependent individuals performing dot probe tasks (Constantinou et al., 2010; Lubman et al., 2000; Marissen et al., 
2006). Recently, our lab reported the first evidence of an AB toward prescription opioids (i.e., opioid $\mathrm{AB}$ ) among chronic pain patients meeting criteria for opioid dependence (Garland et al., 2013b). In this study, opioid-dependent chronic pain patients exhibited significantly greater opioid $\mathrm{AB}$ than chronic pain patients who used, but were not dependent on, opioids. In addition, opioid AB was significantly positively correlated with opioid craving. Although this study established the presence of an opioid AB, it is not known whether opioid AB predicts future opioid misuse. Hence, the purpose of the present study was to examine opioid $\mathrm{AB}$ as a potential predictor of opioid misuse among chronic pain patients. We hypothesized that patients with higher levels of opioid AB at initial evaluation would evidence greater risk for future opioid misuse than patients with low levels of opioid $\mathrm{AB}$, even after controlling for other factors including initial levels of opioid misuse risk, pain severity, and opioid dependence diagnosis. Because exposure to opioid cues might elicit craving responses in addition to attentional responses, we also examined cue-elicited craving as a predictor of future opioid misuse risk.

\section{METHODS}

Data for this study were drawn from a randomized controlled trial that compared the efficacy of an eight-week long mindfulness-based treatment, Mindfulness-Oriented Recovery Enhancement (MORE), to that of a support group (SG) control condition (random allocation ratio 1:1) vis-a-vis reduction of chronic pain and opioid misuse (Garland et al., 2014b). Participants were recruited from primary care, pain, and neurology clinics, reported recurrent pain on more days than not stemming from chronic non-cancer-related pain conditions, and had taken opioid analgesics daily or nearly every day for at least the past 90 days (Chou et al., 2009). At pretreatment, participants completed self-report measures of pain severity and the Current Opioid Misuse Measure (COMM; Butler et al., 2007), and a dot probe task designed to measure opioid AB. At a 3-month posttreatment follow-up, participants completed the COMM again. Participants with full opioid AB and opioid misuse data ( $\mathrm{N}=47$; $64 \%$ female; aged $48 \pm 13$ years) were included in the present study. The study protocol was approved by the Florida State University Institutional Review Board, and all procedures complied with the 1975 Helsinki Declaration. Subjects provided informed consent for their participation.

Participants were assessed for prescription opioid dependence with the Mini-International Neuropsychiatric Interview 6.0 (MINI; Sheehan et al., 1998), and were excluded if suicidal or psychotic. Although all participants reported symptoms of physiological dependence (i.e., tolerance and withdrawal) on the MINI resulting from prolonged use of opioids, a smaller percentage (30\%) met DSM-IV opioid use disorder criteria. However, most (73\%) participants reported opioid analgesic misuse at pretreatment as defined by a validated cutpoint on the COMM (Meltzer et al., 2011). A number of subjects met diagnostic criteria for comorbid major depression (61\%) and/or generalized anxiety disorder (30\%). Participants were paid \$200 for study participation.

\subsection{Measures}

2.1.1 Dot probe task-A dot probe task was used to measure opioid AB. This task was generated in E-Prime 2.0 (PST Inc., Pittsburgh, PA) and presented on 15" computer monitor. 
Each trial began with a fixation cross presented for $500 \mathrm{~ms}$. Next, two images matched for visual complexity, composition, and figure-ground relationships appeared side by side on the screen. On critical trials, pairs of photos containing one opioid-related image and one neutral image were presented for either 200 or $2000 \mathrm{~ms}$. On filler trials, pairs of neutral photos were presented. The set of 12 opioid images represented commonly prescribed opioids in a number of forms, including photos of pills (e.g., Oxycontin), pill bottles, and powdered opioids for insufflation. Neutral images included 12 photos culled from the International Affective Picture System (Lang et al., 1997) depicting household items (e.g., napkin, pencil). Presentation duration and left/right position of the images were randomized and counterbalanced across 12 filler trials and 64 critical trials. A target probe (one dot) replaced one of the images after a $50 \mathrm{~ms}$ inter-stimulus interval (ISI). Probes appeared for $100 \mathrm{~ms}$ and their locations were counterbalanced. Participants indicated probe location via a button press on a keypad.

2.1.2 Cue-elicited craving - Immediately before and after the opioid dot probe task participants were asked "How much do you want your opioids right now?" anchored on a 10-point scale $(1=$ not at all, $10=$ extremely). The difference of these ratings was used to index cue-elicited craving.

2.1.3 Opioid misuse risk—The Current Opioid Misuse Measure (COMM; $\alpha=.83$; Butler et al., 2007) assessed aberrant drug-related behavior. Participants responded to 17 items rated on a Likert scale $(0=$ never, $4=$ very often $)$ regarding how often in the past 30 days they had engaged in behaviors potentially reflective of opioid misuse or took opioid medication in excessive doses or in nonprescribed ways. A validated cut-point of 13 was used to establish whether or not a participant could be classified at high risk for opioid misuse (Meltzer et al., 2011).

2.1.4 Pain severity-Pain severity was measured with the 4-item Pain Severity subscale of the Brief Pain Inventory (BPI; $a=.87$ ) widely used to assess chronic pain (Cleeland, 1994). Response options ranged from 0 (no pain) to 10 (pain as bad as I can imagine).

\subsection{Data Analysis}

With regard to the analysis of participant $\mathrm{AB}$ data, trials with extreme RTs, defined as those with RTs \pm 3 SDs beyond the individual mean RT (Field et al., 2004; Ratcliffe, 1993), were eliminated as outliers $(<2 \%)$. Trials on which the probe location was incorrectly identified (13\%) were also omitted (Townshend and Duka, 2007). AB scores were calculated by subtracting participant's mean RT to probes replacing opioid photos from their mean RT to probes replacing neutral photos, such that positive scores indicate an $\mathrm{AB}$ toward opioid cues (Field et al., 2004). AB scores were approximately normally distributed (KolmogorovSmirnov p's > .10).

Hierarchical linear regression analyzed to what extent pretreatment opioid $\mathrm{AB}$ scores and cue-elicited craving predicted opioid misuse at 3-months posttreatment, after controlling for treatment condition (MORE vs. SG) and pretreatment opioid dependence diagnosis, opioid misuse, and pain severity. Collinearity diagnostics were assessed and no predictor variables 
exceeded recommended tolerance $(<0.2)$ and variance inflation factor thresholds $(>5.0)$.

Stepwise and logistic regression analyses were used for sensitivity analyses.

\section{RESULTS}

\subsection{Between-groups differences in opioid AB}

In unadjusted analyses, individuals exceeding the validated cut-point on the COMM for high risk of opioid misuse at 3-months follow-up had significantly higher $200 \mathrm{~ms}(t=2.15, p=$. $035, d=.66)$ and $2000 \mathrm{~ms}$ opioid $\mathrm{AB}$ scores $(t=2.16, p=.037, d=.68)$ at pretreatment than individuals scoring beneath the cut-point on the COMM (see Figure 1). Within-group onesample t-tests revealed that those exceeding the cut-point on the COMM at 3-months followup had significantly nonzero mean $200 \mathrm{~ms}$ opioid $\mathrm{AB}(t=2.69, p=.012)$, but did not have significantly nonzero mean $2000 \mathrm{~ms}$ opioid $\mathrm{AB}(t=1.63, p=.11)$. For individuals scoring beneath the cut-point on the COMM at follow-up, $2000 \mathrm{~ms} \mathrm{AB}(t=1.88, p=.07)$ and 200 ms opioid $\mathrm{AB}(t=-.32, p=.75)$ scores were not significantly different than zero. Because the 2000 ms opioid AB was not significantly different than zero for either group, this variable was not included in subsequent regression analyses.

\subsection{Prediction of future opioid misuse following treatment}

In step 1, pretreatment COMM scores explained a significant portion of variance in COMM scores at 3-months follow-up, irrespective of treatment condition, pre-treatment pain severity, and presence of opioid dependence diagnosis. In step 2, adding cue-elicited craving and $200 \mathrm{~ms}$ opioid AB scores to the model significantly predicted future COMM scores and contributed a significant increase in Model $R^{2}$. The full model predicted $50 \%$ of the variance in COMM scores at 3-month follow-up (see Table 1). In supplementary analyses ${ }^{1}$, we conducted a stepwise regression with removal. Model results did not differ substantively in terms of valence or statistical significance from the hierarchical regression described above: baseline opioid misuse, pain severity, cue-elicited craving, and $200 \mathrm{~ms}$ opioid AB were retained in the final stepwise model. Logistic regression analysis predicting whether or not participants exceeded the opioid misuse threshold on the COMM at 3-month follow-up found this final set of variables to correctly classify $89.3 \%$ of cases.

\section{DISCUSSION}

Findings from this study of chronic pain patients treated for comorbid pain and prescription opioid misuse suggest that attentional and subjective reactivity to opioid cues prior to behavioral treatment may signal future risk of opioid misuse. Consistent with our hypotheses, above and beyond the predictive power of pre-treatment levels of opioid misuse risk, pain severity, and prescription opioid dependence, $200 \mathrm{~ms}$ opioid AB scores and cueelicited craving significantly and independently predicted risk of opioid misuse more than 20 weeks later. To our knowledge, this is the first study to demonstrate that $\mathrm{AB}$ to prescription opioids prospectively predicts opioid misuse following treatment. The combined predictive power of opioid $\mathrm{AB}$ and cue-elicited craving (which together explained

\footnotetext{
${ }^{1}$ Supplementary material can be found by accessing the online version of this paper at http://dx.doi.org and by entering doi:...
} 
$17 \%$ of the variance in opioid misuse risk at 3-months posttreatment) indicates that these factors may be markers of the proclivity to engage in opioid misuse.

In unadjusted analyses, individuals at elevated risk for opioid misuse at 3-months posttreatment follow-up exhibited a significant mean AB toward opioid cues presented for $200 \mathrm{~ms}$, whereas those at low risk for opioid misuse had a nonsignificant bias away from opioid cues presented for $2000 \mathrm{~ms}$ (possibly indicating attentional avoidance of drug stimuli; e.g., Garland et al., 2012b). In individual difference analyses adjusted for clinically relevant covariates, $200 \mathrm{~ms}$ AB scores significantly predicted future opioid misuse risk. The $200 \mathrm{~ms}$ $\mathrm{AB}$ is theorized to index automatic, initial orienting toward drug cues, whereas $\mathrm{AB}$ for longer duration stimuli (ranging from $500-2000 \mathrm{~ms}$ ) is theorized to index higher-order attentional processes, such as delayed disengagement of attention from drug-related cues (Field and Cox, 2008). Thus, it appears as if opioid misusers exhibit initial attentional orienting toward opioid cues prior to treatment and this attentional orienting independently predicts the tendency to engage in opioid misuse in multivariate analyses controlling for factors like pre-treatment opioid misuse, prescription opioid dependence diagnosis, and chronic pain severity. Furthermore, heightened cue-elicited craving during the dot probe task predicted future opioid misuse risk, indicating that individuals who experienced the largest increases in craving following exposure to opioid cues were most at-risk to misuse opioids 20 weeks later.

This study was limited by the lack of a quantitative measure of opioid dosing and adjuvant psychiatric medication use. Future studies should carefully assess opioid misuse by tracking opioid dosing via prescription history, pill count, and toxicology screens, as well as through clinician-rated opioid misuse assessment and legal history. Despite these limitations, study findings suggest that behavioral and self-reported indices of opioid reactivity may reliably signal future opioid misuse risk following treatment, and therefore provide potential prognostic indicators of treatment outcome. Interventions that target opioid AB and cuereactivity hold promise for the treatment of opioid misuse among chronic pain patients (Garland et al., 2014a).

\section{Supplementary Material}

Refer to Web version on PubMed Central for supplementary material.

\section{Acknowledgments}

Role of the Funding Source: This work was supported by grant numbers DA032517 and DA037005 from the National Institutes of Health awarded to ELG.; and a grant from the Fahs Beck Fund for Research and Experimentation, also awarded to ELG. NIDA and the Fahs Beck Fund had no further role in study design; in the collection, analysis and interpretation of data; in the writing of the report; or in the decision to submit the paper for publication.

\section{References}

Becker S, Gandhi W, Schweinhardt P. Cerebral interactions of pain and reward and their relevance for chronic pain. Neurosci Lett. 2012; 520:182-187. [PubMed: 22440855]

Besson JM. The neurobiology of pain. Lancet. 1999; 353:1610-1615. [PubMed: 10334274] 
Butler SF, Budman SH, Fernandez KC, Houle B, Benoit C, Katz N, Jamison RN. Development and validation of the Current Opioid Misuse Measure. Pain. 2007; 130:144-156. [PubMed: 17493754]

Chou R, Fanciullo GJ, Fine PG, Adler JA, Ballantyne JC, Davies P, Donovan MI, Fishbain DA, Foley $\mathrm{KM}$, Fudin J, et al. Clinical guidelines for the use of chronic opioid therapy in chronic noncancer pain. J Pain. 2009; 10:113-130. [PubMed: 19187889]

Cleeland, CS. Brief Pain Inventory-Short Form (BPI-SF). MD Anderson Cancer Center; Houston, TX: 1994.

Constantinou N, Morgan CJ, Battistella S, O'Ryan D, Davis P, Curran HV. Attentional bias, inhibitory control and acute stress in current and former opiate addicts. Drug Alcohol Depend. 2010; 109:220225. [PubMed: 20172662]

Cox WM, Hogan LM, Kristian MR, Race JH. Alcohol attentional bias as a predictor of alcohol abusers' treatment outcome. Drug Alcohol Depend. 2002; 68:237-243. [PubMed: 12393218]

Field M, Cox WM. Attentional bias in addictive behaviors: a review of its development, causes, and consequences. Drug Alcohol Depend. 2008; 97:1-20. [PubMed: 18479844]

Field M, Mogg K, Zetteler J, Bradley BP. Attentional biases for alcohol cues in heavy and light social drinkers: the roles of initial orienting and maintained attention. Psychopharmacology (Berl). 2004; 176:88-93. [PubMed: 15071718]

Field M, Munafo MR, Franken IH. A meta-analytic investigation of the relationship between attentional bias and subjective craving in substance abuse. Psychol Bull. 2009; 135:589-607. [PubMed: 19586163]

Fishbain DA, Cole B, Lewis J, Rosomoff HL, Rosomoff RS. What percentage of chronic nonmalignant pain patients exposed to chronic opioid analgesic therapy develop abuse/addiction and/or aberrant drug-related behaviors? A structured evidence-based review. Pain Med. 2007; 9:444-459. [PubMed: 18489635]

Franken IH. Drug craving and addiction: integrating psychological and neuropsychopharmacological approaches. Prog Neuropsychopharmacol Biol Psychiatry. 2003; 27:563-579. [PubMed: 12787841]

Garland EL, Franken IH, Howard MO. Cue-elicited heart rate variability and attentional bias predict alcohol relapse following treatment. Psychopharmacology (Berl). 2012a; 222:17-26. [PubMed: 22203318]

Garland EL, Franken IH, Sheetz JJ, Howard MO. Alcohol attentional bias is associated with autonomic indices of stress-primed alcohol cue-reactivity in alcohol-dependent patients. Exp Clin Psychopharmacol. 2012b; 20:225-235. [PubMed: 22329555]

Garland EL, Froeliger B, Howard MO. Effects of Mindfulness-Oriented Recovery Enhancement on reward responsiveness and opioid cue-reactivity. Psychopharmacology (Berl). 2014a; 231:3229_ 3238. [PubMed: 24595503]

Garland EL, Froeliger B, Zeidan F, Partin K, Howard MO. The downward spiral of chronic pain, prescription opioid misuse, and addiction: cognitive, affective, and neuropsychopharmacologic pathways. Neurosci Biobehav Rev. 2013a; 37:2597-2607. [PubMed: 23988582]

Garland EL, Froeliger BE, Passik SD, Howard MO. Attentional bias for prescription opioid cues among opioid dependent chronic pain patients. J Behav Med. 2013b; 36:611-620. [PubMed: 22968666]

Garland EL, Manusov EG, Froeliger B, Kelly A, Williams JM, Howard MO. Mindfulness-oriented recovery enhancement for chronic pain and prescription opioid misuse: results from an early-stage randomized controlled trial. J Consult Clin Psychol. 2014b; 82:448-459. [PubMed: 24491075]

Lang, P.; Bradley, M.; Cuthbert, B. NIMH Center for the Study of Emotion and Attention. University of Florida; 1997. International Affective Picture System (IAPS): Technical Manual And Affective Ratings.

Le Bars D, Guilbaud G, Chitour D, Besson JM. Does systemic morphine increase descending inhibitory controls of dorsal horn neurones involved in nociception? Brain Res. 1980; 202:223228. [PubMed: 6253026]

Lubman DI, Peters LA, Mogg K, Bradley BP, Deakin JF. Attentional bias for drug cues in opiate dependence. Psychol Med. 2000; 30:169-75. [PubMed: 10722187] 
Marissen MA, Franken IH, Waters AJ, Blanken P, van den Brink W, Hendriks VM. Attentional bias predicts heroin relapse following treatment. Addiction. 2006; 101:1306-1312. [PubMed: 16911730]

Meltzer EC, Rybin D, Saitz R, Samet JH, Schwartz SL, Butler SF, Liebschutz JM. Identifying prescription opioid use disorder in primary care: diagnostic characteristics of the Current Opioid Misuse Measure (COMM). Pain. 2011; 152:397-402. [PubMed: 21177035]

Oertel BG, Preibisch C, Wallenhorst T, Hummel T, Geisslinger G, Lanfermann H, Lötsch J. Differential opioid action on sensory and affective cerebral pain processing. Clin Pharmacol Ther. 2007; 83:577-588. [PubMed: 18030306]

Pierce RC, Vanderschuren L. Kicking the habit: The neural basis of ingrained behaviors in cocaine addiction. Neurosci Biobehav Rev. 2010; 35:212-219. [PubMed: 20097224]

Ratcliffe R. Methods of dealing with reaction-time outliers. Psychol Bull. 1993; 114:510-532. [PubMed: 8272468]

Robinson TE, Berridge KC. Incentive-sensitization and addiction. Addiction. 2001; 96:103-14. [PubMed: 11177523]

Sheehan DV, Lecrubier Y, Sheehan KH, Amorim P, Janavs J, Weiller E, Hergueta T, Baker R, Dunbar GC. The Mini-International Neuropsychiatric Interview (M.I.N.I.): the development and validation of a structured diagnostic psychiatric interview for DSM-IV and ICD-10. J Clin Psychiatry. 1998; 59(Suppl 20):22-33. quiz 34-57. [PubMed: 9881538]

Shippenberg TS, Bals-Kubik R, Herz A. Examination of the neurochemical substrates mediating the motivational effects of opioids: role of the mesolimbic dopamine system and D-1 vs. D-2 dopamine receptors. J Pharmacol Exp Ther. 1993; 265:53-59. [PubMed: 8386244]

Stacy AW, Wiers RW. Implicit cognition and addiction: a tool for explaining paradoxical behavior. Ann Rev Clin Psychol. 2010; 6:551-575. [PubMed: 20192786]

Tiffany ST. A cognitive model of drug urges and drug-use behavior: role of automatic and nonautomatic processes. Psychol Rev. 1990; 97:147-168. [PubMed: 2186423]

Townshend JM, Duka T. Avoidance of alcohol-related stimuli in alcohol-dependent inpatients. Alcohol Clin Exp Res. 2007; 31:1349-1357. [PubMed: 17550367]

Wagner KJ, Sprenger T, Kochs EF, Tölle TR, Valet M, Willoch F. Imaging human cerebral pain modulation by dose-dependent opioid analgesia: a positron emission tomography activation study using remifentanil. Anesthesiology. 2007; 106:548-556. [PubMed: 17325514]

Wilson JF. Strategies to stop abuse of prescribed opioid drugs. Ann Intern Med. 2007; 146:897-900. [PubMed: 17577013]

Wise RG, Rogers R, Painter D, Bantick S, Ploghaus A, Williams P, Rapeport G, Tracey I, et al. Combining fMRI with a pharmacokinetic model to determine which brain areas activated by painful stimulation are specifically modulated by remifentanil. Neuroimage. 2002; 16:999. [PubMed: 12202088]

Yaksh TL. Spinal opiate analgesia: characteristics and principles of action. Pain. 1981; 11:293-346. [PubMed: 6276842]

Yaksh TL. Pharmacology of spinal adrenergic systems which modulate spinal nociceptive processing. Pharmacol Biochem Behav. 1985; 22:845-858. [PubMed: 2861606]

Drug Alcohol Depend. Author manuscript; available in PMC 2015 November 01. 
- Opioid dependent pain patients exhibit an attentional bias toward opioid cues

- Opioid attentional bias predicted opioid misuse 20 weeks later

- Cue-elicited craving predicted opioid misuse 20 weeks later

- Attentional bias and cue-elicited craving may signal opioid misuse risk 


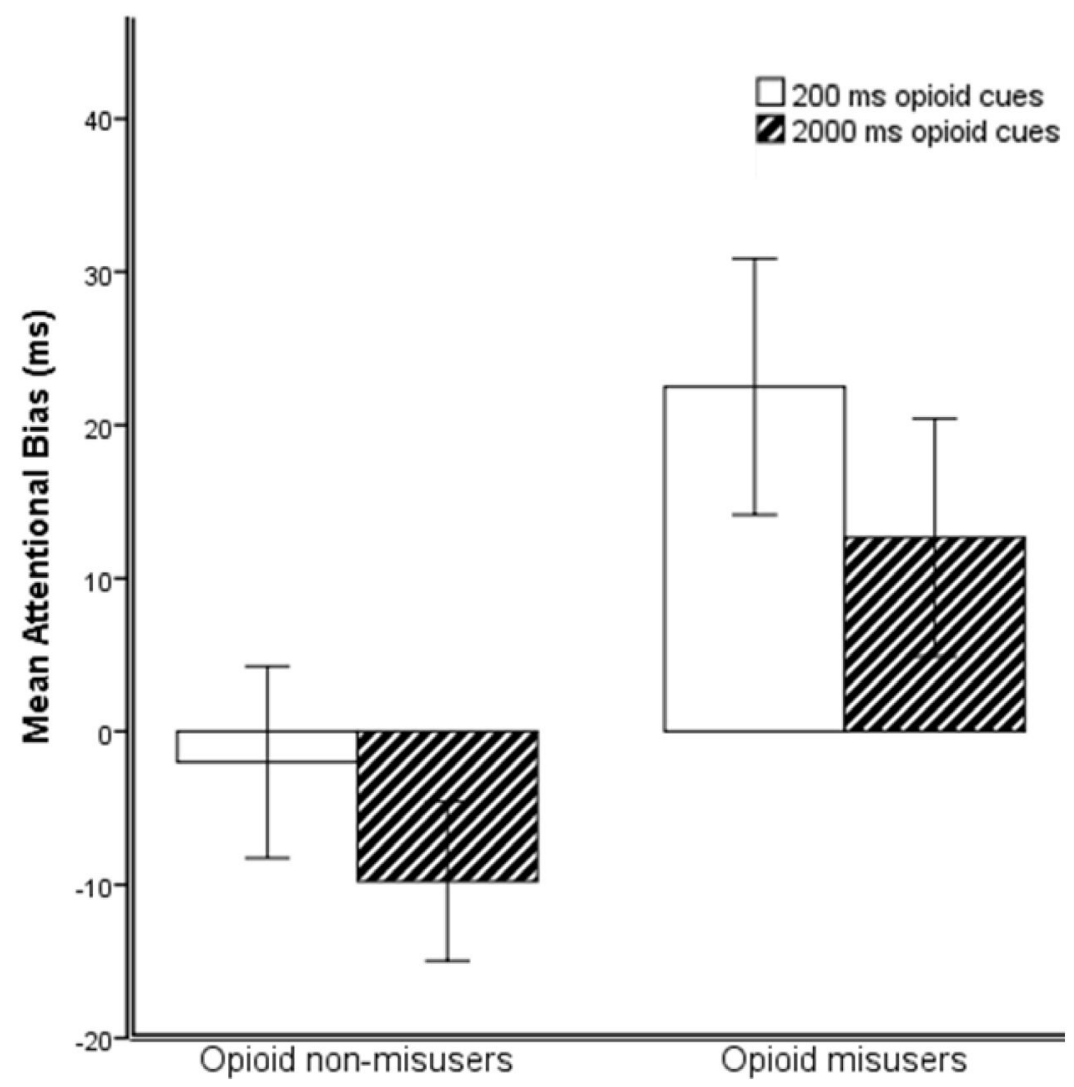

Figure 1.

Between-groups differences in pre-treatment opioid attentional bias scores (higher number = greater $A B)$ for patients with elevated opioid misuse risk $(n=28)$ and low risk for opioid misuse $(n=19) 3$ months following completion of behavioral treatment for chronic pain and opioid misuse. Error bars indicate \pm 1 standard error of measurement. 


\section{Table 1}

Predictors of opioid misuse risk at 3-months posttreatment for a sample of chronic patient patients completing behavioral treatments.

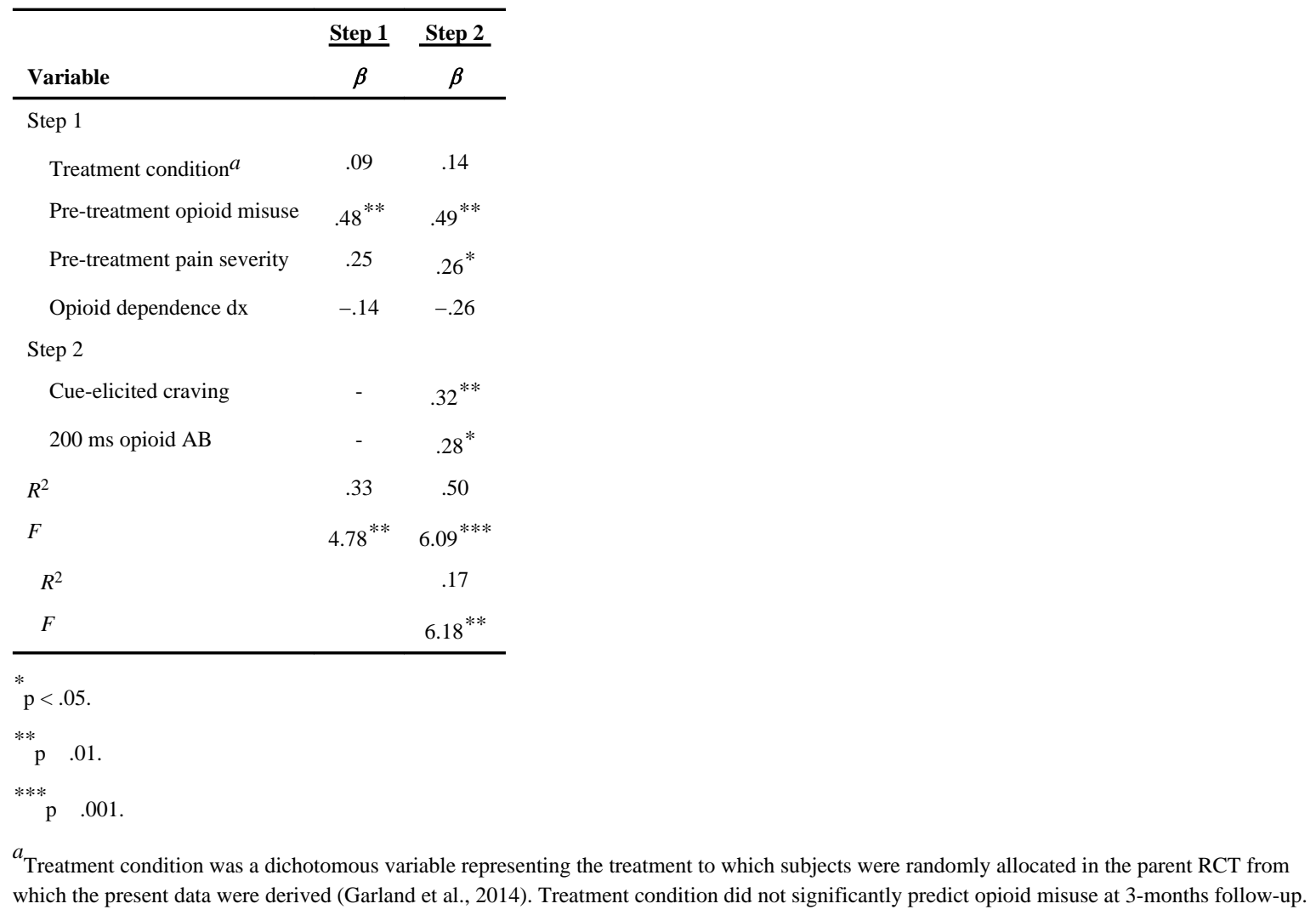

